\title{
A Woman Cannot Die from a Pregnancy She Does Not Have
}

More than $99 \%$ of maternal deaths* occur in low-resource settings; in parts of the developing world, as many as one in six women die of maternal causes. ${ }^{1}$ Currently, seven developing countries-Afghanistan, Angola, Malawi, Niger, Rwanda, Sierra Leone and Tanzania-have maternal mortality ratios of at least 1,400 maternal deaths per 100,000 live births. ${ }^{2}$

Levels of maternal mortality were similarly high in Europe and North America only a century ago; today, however, a woman's lifetime risk of dying from maternal causes is as low as one in 30,000 in these areas (e.g., the MMR in Sweden in 2005 was 12/100,000). 1,3,4 Much of this decline in maternal mortality has resulted from improvements in obstetric care (including early recognition of risk factors for pregnancy complications), access to safe abortion and other advances in health care. ${ }^{l}$

Such improvements in obstetric care are urgently needed in the developing world-for example, all women should have a skilled birth attendant and access to medical facilities-and would do much to reduce the high rates of maternal death. However, a commitment to complex, high-cost, long-term solutions to the problem of maternal mortality should not divert us from immediate, low-cost, low-tech approaches. ${ }^{5}$ One such solution is simply reducing fertility.

Fertility has declined during the last century, particularly in industrialized countries, 5,6,7 and this decline has accompanied changes in family structure and desired family size, urbanization, and increased access to and use of modern contraceptives. However, in many developing regions where maternal mortality is high, fertility remains high as well. The total fertility rate (TFR)-the average number of live births a woman has during her lifetime-is still at least five children per woman in 34 countries and territories, most of them in Sub-Saharan Africa. ${ }^{2}$

In Prata and colleagues' 2010 cost-benefit analysis of different aspects of maternal care, the combination of two reproductive health interventions that affect fertility ratesproviding family planning and providing access to safe abortion-was the most economical way to reduce maternal deaths in low-resource settings. ${ }^{8}$ However, two questions remain: Exactly what role do fertility decline and im-

\footnotetext{
*The World Health Organization defines maternal death as "the death of a woman while pregnant or within 42 days of termination of pregnancy, irrespective of the duration and site of the pregnancy, from any cause related to or aggravated by the pregnancy or its management but not from accidental or incidental causes." (source: World Health Organization, Health statistics and health information systems, <http://www.who.int/ healthinfo/statistics/indmaternalmortality/en/index.html>, accessed July $25,2011$.
}

provements in obstetric care play in reducing maternal mortality? And, which intervention decreases maternal mortality most effectively?

\section{A Hypothetical Exercise}

To highlight the role of fertility decline in reducing maternal deaths, we conducted an exercise using historical data on fertility and maternal mortality to create two hypothetical scenarios. In the first scenario, fertility levels in a population stayed the same between the early 1900s and the early 2000s, while maternal mortality ratios fell to 2005 levels. This scenario estimated the impact of improved obstetric care on maternal mortality. In the second scenario, we reversed the situation, using century-old maternal mortality rates in combination with current TFRs; this allowed us to estimate how reducing fertility affects the number of maternal deaths.

Our analysis focused on Sweden, one of the few countries for which long-term, high-quality, age-specific data on fertility and maternal mortality ratios are available. We used data from 1911 and 2005 (or as close to these years as possible) for women aged 15-49. Because maternal mortality is rare in Sweden (in 2005, only six women died of causes related to pregnancy or childbirth ${ }^{9}$ ), we could not calculate age-specific maternal mortality ratios for 2005. Therefore, we used Wildman and colleagues' $2004^{4}$ estimates of age-specific maternal mortality ratios for European countries with low maternal mortality. The larger population size of the pooled multicountry data set allowed for more precise and accurate ratios for each agegroup, and we feel confident that these are reasonable estimates for Sweden.

Given the nature of our exercise, it is not necessary to have rates that are only from Sweden, or that are exactly from 2005. For simplicity of comparison, as well as to control for differences in the age distribution of the population between 1911 and 2005, we created a standard population size by applying the World Health Organization's (WHO) Scandinavian Standard Population Distribution ${ }^{10}$ to a fictional population of 100 million. This population size was chosen to make the number of deaths under each scenario a whole number. The $\mathrm{WHO}$ age distributions represented both males and females; we assumed that $50 \%$ of the population was female, yielding a population of 50 million women, of whom 25 million were aged 15-49 (Table 1 , page 156).

For each age-group in our hypothetical scenarios, we estimated the number of births by multiplying the age-

\section{By Nadia \\ Diamond-Smith and Malcolm Potts}

Nadia DiamondSmith is a doctoral candidate in the Department of Population, Family and Reproductive Health, Johns Hopkins Bloomberg School of Public Health, Baltimore, MD, USA. Malcolm Potts is professor and Bixby Chair, Bixby Center for Population, Health and Sustainability, University of California, Berkeley, USA. 


\begin{tabular}{|c|c|c|c|c|c|c|c|}
\hline \multirow{2}{*}{$\begin{array}{l}\text { Age- } \\
\text { group }\end{array}$} & \multirow{2}{*}{$\begin{array}{l}\text { Standard* } \\
\text { population } \\
\text { size (in 000s) }\end{array}$} & \multicolumn{3}{|c|}{ Age-specific fertility rate } & \multicolumn{3}{|c|}{ Maternal mortality ratio } \\
\hline & & 1911 & 2005 & $\%$ reduction & 1911 & 2005 & $\%$ reduction \\
\hline $15-19$ & 4,000 & 0.10 & 0.03 & 70.00 & 1,136 & 8.4 & 99.26 \\
\hline $20-29$ & 7,000 & 1.44 & 0.78 & 45.83 & 971 & 6.31 & 99.35 \\
\hline $30-39$ & 7,000 & 1.54 & 0.91 & 40.91 & 1,263 & 9.14 & 99.28 \\
\hline 40-49 & 7,000 & 0.40 & 0.05 & 87.50 & 2,190 & 23.76 & 98.91 \\
\hline Total & 25,000 & 3.48 & 1.77 & 49.14 & 1,390 & 11.9 & 99.14 \\
\hline
\end{tabular}

*To control for differences in the age distribution of the population between 1911 and 2005, we created a standard population size by applying the World Health Organization's (WHO) Scandinavian Standard Population Distribution to a fictional population of 100 million. This population size was chosen to make the number of deaths under each scenario a whole number. The WHO age distributions represented both males and females; we assumed that $50 \%$ of the population was female,yielding a population of 50 million women, of whom 25 million were aged 15-49. Notes:Age-specific fertility rate is the number of births to women in a specific age-group, per the number of women in that age-group. Maternal mortality ratio is number of maternal deaths per 100,000 live births. Sources: Standard population size-reference 10. Total fertility rate-reference 7. Maternal mortality ratio-1911:reference 3;2005: reference 4.

specific total fertility rate (obtained from the Human Fertility Database ${ }^{7}$ ) by the number of women, and then dividing by the number of years in the age-group. We added the TFRs for the four age-groups to obtain the total fertility rate for the entire population. The number of maternal deaths in each age-group was then calculated by multiplying the age-specific maternal mortality ratio (maternal deaths per 100,000 births) by the number of births in the group. All women in each age-group and time period were considered to have reproductive potential.

Through these calculations, we estimated that in our standard population, more than 30,000 maternal deaths would occur if the TFR and maternal mortality ratio were both at 1911 levels (Table 2). Changing both measures to their 2005 values reduced the number of deaths to 104 . Improving obstetric care alone, so that the maternal mortality ratio declined to the 2005 level but fertility remained at its 1911 value (scenario 1), had a larger impact on the number of maternal deaths than did reducing only the fertility rate (scenario 2); nevertheless, fertility decline alone halved the number of maternal deaths, to fewer than 15,000

Reducing fertility disproportionally affected maternal mortality in the high-risk age-groups (i.e., the youngest and oldest women). For example, the maternal mortality rate for the youngest women (15-19 years), who face a higher risk for adverse outcomes, fell by two-thirds (69\%), but among 20-29-year-old women (those at lower risk), it

\begin{tabular}{|c|c|c|c|c|}
\hline Age-group & $\begin{array}{l}1911 \text { TFR/ } \\
1911 \mathrm{MMR}\end{array}$ & $\begin{array}{l}2005 \text { TFR/ } \\
2005 \text { MMR }\end{array}$ & $\begin{array}{l}1911 \mathrm{TFR} / \\
2005 \mathrm{MMR} \\
\text { (scenario 1) }\end{array}$ & $\begin{array}{l}2005 \text { TFR/ } \\
1911 \text { MMR } \\
\text { (scenario 2) }\end{array}$ \\
\hline $15-19$ & 894 & 2 & 7 & 279 \\
\hline $20-29$ & 9,822 & 34 & 64 & 5,312 \\
\hline 30-39 & 13,573 & 58 & 98 & 8,011 \\
\hline $40-49$ & 6,169 & 9 & 67 & 836 \\
\hline Total & 30,458 & 104 & 235 & 14,437 \\
\hline
\end{tabular}

Notes: Numbers may not sum to total because of rounding. TFR=total fertility rate. $\mathrm{MMR}=$ maternal mortality ratio. declined by less than half (46\%, Table 3). Overall, change in the TFR alone was sufficient to reduce maternal mortality by 53\%. In other words, had obstetric care not improved in the past century, fertility decline by itself would have left Sweden with half as many maternal deaths in 2005 as it had in 1911. The decline in maternal mortality ratios to 2005 levels did not differentially affect the most at-risk age-groups: All groups experienced a 99\% decline in maternal mortality.

\section{Reducing Fertility Works}

This exercise is purely hypothetical, but its strength lies in its simplicity. Although we used historical data from Sweden, rather than from the developing world, our analysis was meant to illustrate a point, rather than to make exact calculations. We believe that the benefits of reducing fertility (through improved access to contraceptives and other means) can be generalized to countries where maternal mortality ratios remain tragically high.

Stover and Ross conducted a sophisticated analysis of the impact of fertility decline on maternal mortality in developing countries, focusing mainly on increased contraceptive use. ${ }^{11}$ According to their analysis, one million fewer maternal deaths occurred between 1990 and 2005 than would have occurred if the fertility rate had remained constant. Most of the decline in mortality occurred in high-risk age-groups. These findings support the argument that fertility decline, unaided, can substantially reduce maternal deaths.

We are not suggesting that improvements in obstetric care have been unimportant in lowering maternal mortality, or that declines in maternal mortality have been achieved without the health improvements of the past century (e.g., improved nutrition and preventive care). However, our results suggest that the relatively modest fertility decline (from 3.5 to 1.8 children per women) that occurred in Sweden during the last century would have produced a sizeable reduction (by approximately 50\%) in the number of maternal deaths, even if improvements in obstetric care had not occurred. Given the high fertility and maternal mortality rates in many developing countries (most of which are in Africa), it is likely that fertility declines could have a more substantial impact on reducing maternal deaths than they did in our exercise using Swedish data.

In most parts of the world, ${ }^{12}$ women and families, when given the opportunity and means to control their fertility, overwhelmingly have chosen to have fewer children; thus, fertility has decreased. The declines in fertility have been largest among the youngest and oldest women, suggesting that family planning has been used mostly by women in the highest-risk age-groups. Therefore, family planning and resulting declines in fertility make a disproportional impact on maternal mortality. ${ }^{11}$ Some studies have found that a large proportion of maternal deaths in developing countries result from induced abortions; increasing access to family planning could prevent many of these deaths, ${ }^{5}$ 


\begin{tabular}{|c|c|c|}
\hline Age-group & $\begin{array}{l}1911 \text { TFR/ } \\
2005 \text { MMR } \\
\text { (scenario 1) }\end{array}$ & $\begin{array}{l}2005 \text { TFR/ } \\
1911 \mathrm{MMR} \\
\text { (scenario 2) }\end{array}$ \\
\hline 15-19 & 99.2 & 68.8 \\
\hline $20-29$ & 99.3 & 45.9 \\
\hline 30-39 & 99.3 & 41.0 \\
\hline 40-49 & 98.9 & 86.4 \\
\hline Total & 99.2 & 52.6 \\
\hline
\end{tabular}

Notes: TFR=total fertility rate.MMR=maternal mortality ratio.

given that $40 \%$ of all pregnancies are unintended and $20 \%$ end in abortion. Women around the world use family planning to space pregnancies, which reduces family size. In addition, increased awareness of and access to family planning also help women and couples to recognize that limiting family size and spacing births are options. ${ }^{13}$ These fundamental changes in family structure not only can lead to improvements in maternal and child health but generate a positive feedback loop: Lower fertility and pregnancy spacing improve women's status by increasing their opportunities for labor force participation; improved status leads to further reductions in desired family size; and smaller family size leads to continued fertility declines and reduced maternal mortality. ${ }^{12}$

The fifth Millennium Development Goal ${ }^{14}$ has brought critical attention to the unacceptably high burden of maternal mortality and the need to improve antenatal health care. However, many of the approaches to reducing maternal mortality (e.g., increasing the number of deliveries at health facilities with skilled attendants or improving access to emergency obstetric care) are complex and will take time to implement. In the meantime, maternal mortality can be reduced relatively inexpensively by preventing unwanted pregnancy through family planning. ${ }^{8}$ The decision to practice family planning is personal and private, and it need not require professionals or health clinics. Although inexpensive at the program level, however, family planning may be difficult for individuals to afford. Thus, women face barriers, including cost, lack of transportation and the fear of side effects (real or rumored). ${ }^{13}$ In developing countries, making contraceptives available and accessible may be the most important, cost-effective and easily accomplished primary health care goal. ${ }^{8}$ Re- ducing barriers to family planning may lessen the burden of maternal death in low-resource settings.

\section{REFERENCES}

1. Ronsmans C and Graham W, Maternal mortality: who, when, where and why, Lancet, 2006, 368(9542):1189-1200.

2. Population Division of the United Nations Secretariat, 2000-2005, Statistics and indicators on women and men, Table 3b, Maternal mortality and infant mortality, <http://unstats.un.org/unsd/ demographic/products/indwm/tab3b.htm>, accessed July 25, 2011.

3. Högberg U and Wall S, Age and parity as determinants of maternal mortality-impact of their shifting distribution among parturients in Sweden from 1781 to 1980 , Bulletin of the World Health Organization, 1986, 64(1):85-91.

4. Wildman $\mathrm{K}$ and Bouvier-Colle MH, Maternal mortality as an indicator of obstetric care in Europe, BJOG, 2004, 111(2):164-169.

5. Campbell $O$ and Graham W, Strategies for reducing maternal mortality: getting on with what works, Lancet, 2006, 368(9543):12841299.

6. Loudon I, Maternal mortality in the past and its relevance to developing countries today, American Journal of Clinical Nutrition, 2000, 72(Suppl.1):S241-S246

7. Max Planck Institute for Demographic Research and Vienna Institute of Demography, Human fertility database: Sweden-Birth counts, population exposures, rates and summary indicators, <http://www.humanfertility.org/cgi-bin/countrypage.php? country=SWE $>$, accessed July 25, 2011.

8. Prata $\mathrm{N}$ et al., Setting priorities for safe motherhood interventions in resource-scarce settings, 2010, Health Policy, 94(1):1-13.

9. The National Board of Health and Welfare (SOCIALSTYRELSEN), Centre for Epidemiology (Epidemiologiskt Centrum), Sweden, <http://www.socialstyrelsen.se>, accessed July 25, 2011.

10. Ahmad OB et al., Age standardization of rates: a new WHO standard, GPE Discussion Paper Series, Geneva: World Health Organization, 2001, No. 31.

11. Stover J and Ross J, How increased contraceptive use has reduced maternal mortality, Maternal and Child Health Journal, 2010, 14(5): 687-695.

12. Cleland J et al., Family planning: the unfinished agenda, Lancet, 2006, 368(9549):1810-1827.

13. Campbell M, Sahin-Hodoglugil NN and Potts M, Barriers to fertility regulation: a review of the literature, Studies in Family Planning, 2006, 37(2):87-98.

14. United Nations, Millennium Development Goals, Goal 5: Improve Maternal Health, <http://www.un.org/millenniumgoals/maternal. shtml $>$, accessed July 29, 2011

\section{Acknowledgments}

The authors would like to thank Kirk Smith, Martha Campbell and Vanja Torbica for their insight into calculations and concept.

Author contact: ndiamond@jhsph.edu 